\title{
Experimental study of high power mm-waves scattering by plasma turbu- lence in TCV plasmas
}

\author{
O. Chellaï ${ }^{1 \star \star}$, S. Alberti ${ }^{1}$, I. Furno ${ }^{1}$, T. Goodman ${ }^{1}$, A. Koehn ${ }^{2}$, L. Figini ${ }^{3}$, D. Ricci ${ }^{3}$, L. Hizanidis ${ }^{4}$, P. Papagiannis ${ }^{4}$, C. \\ Tsironis $^{4}$, and the TCV team ${ }^{1}$ \\ ${ }^{1}$ Swiss Plasma Center, Ecole Polytechnique Fédérale de Lausanne, Switzerland \\ ${ }^{2}$ Max Planck Institute for Plasma Physics, Garching, Germany \\ ${ }^{3}$ Instituto di Fisica del Plasma, CNR, Milan, Italy \\ ${ }^{4}$ National Center for Scientific Research Demokritos, National Technical University of Athens, Greece
}

\begin{abstract}
Understanding the propagation of high power mm-wave in plasmas is of tremendous importance in the route to fusion considering their extensive use in magnetically confined fusion devices. Mm-beams, launched from the outside of the vessel must propagate through plasma edge-turbulence before reaching their target region. Until recently, the effect of edge-turbulence on the beam propagation was neglected, but it has been estimated for ITER that it could lead to significant differences in the time-averaged and instantaneous beam profiles, leading to a loss of efficiency in their use. In this paper, we present first direct experimental measurements of high power beam after propagation in simple magnetized toroidal plasmas in TCV.
\end{abstract}

\section{Introduction}

High power mm-waves are extensively used in tokamaks by locally driving plasma current to stabilize neoclassical tearing modes (NTMs) [1]. A narrow beam is launched from the outside of the vessel, targeting the core plasma. Along its propagation, the mm-beam traverses the highly turbulent Scrape-off Layer (SOL) region. The SOL is localized at the edge of the tokamak plasma and is characterized by large electron density fluctuations (up to $100 \%$ of the background density) associated with field-aligned filaments, commonly referred to as blobs [2]. The inhomogeneity of the electron density is responsible for scattering of the mm-wave. Unlike in present devices, in future large tokamaks, such as ITER, the path length of the beam will be long enough to enable small perturbations in the SOL to cause significant changes in both the time averaged and instantaneous beam profiles. This may lead to its broadening on average [3] and consequently to a loss of efficiency in NTM stabilization [4]. Only recently has the scattering process been investigated for beam propagation estimations in future larger devices like ITER. Analytical model for mm-wave scattering by blobs are developed [5] and state-of-the-art codes are upgraded to account for the effect of edge turbulence [3] [6]. Yet, there is still a need for experimental data to validate the simulation tools and reach predictive capabilities for ITER and beyond.

In this paper, we present first direct poloidal measurements of high power $(\approx 100 \mathrm{~kW})$ third electron cyclotron harmonic beam in the Tokamak à Configuration Variable (TCV) after propagation in a turbulent plasma in a simple magnetized torus (SMT) configuration (see section 2.1).
Electron density measurements in the plasma edge are presented together with measurements of the transmitted mmpower.

\section{Experimental setup}

In this section, we present the experimental setup (Fig. 1). First, the TCV experiment is described together with plasma conditions. Then a detailed presentation of the mm-beam diagnostic is given. The main experimental challenges are mentioned at the end of this section.

\begin{tabular}{cc}
\hline Major radius R & $0.88 \mathrm{~m}$ \\
Minor radius a & $0.25 \mathrm{~m}$ \\
Toroidal field $B_{T}$ & $1.3-1.43 \mathrm{~T}$ \\
Electron density $n_{e}$ & $0.7-4 \times 10^{18} \mathrm{~m}^{-3}$ \\
Second harmonic ECRH (X2) & $82.7 \mathrm{GHz} / 3.6 \mathrm{~mm}$ \\
Third harmonic ECRH (X3) & $117.8 \mathrm{GHz} / 2.5 \mathrm{~mm}$ \\
Total RF power & $550 \mathrm{~kW}$ \\
\hline
\end{tabular}

Table 1. TCV parameters in typical simple magnetized toroidal discharges

\subsection{Tokamak à Configuration Variable (TCV)}

The TCV tokamak (see Table 1.) is characterized by its high shaping capabilities enabled by a plasma control system made of 16 independent shaping coils. TCV possesses a unique $4.5 \mathrm{MW}$ electron cyclotron resonance heating $(\mathrm{ECRH})$ system distributed over two frequencies 


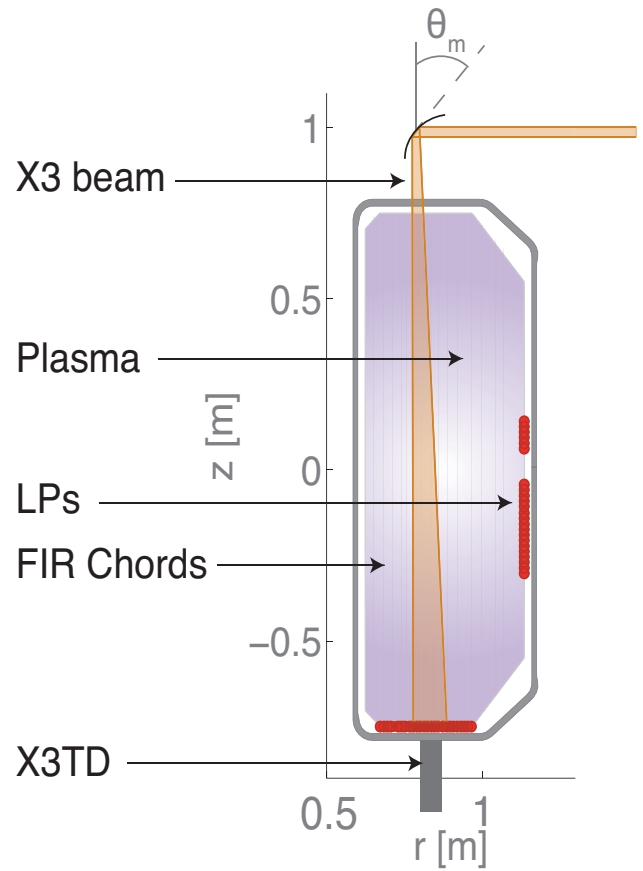

Figure 1. Experimental setup: a mm-beam is injected from the top of the vessel through a steerable mirror and traverses the plasma. The power reaching the floor of the vessel is detected by the X3 transmission diagnostic (X3TD). Line-integrated densities are measured along the vertical chords of the Far Infrared Interferometer (FIR). Plasma edge density fluctuations are measured by wall mounted Langmuir probes.

(second and third harmonic $\mathrm{X}$-mode, respectively $\mathrm{X} 2$ at $82.7 \mathrm{GHz}$ and $\mathrm{X} 3$ at $117.8 \mathrm{GHz}$ ) [7]. The path length of the $\mathrm{X} 3$ beam to the $\mathrm{X} 3$ transmission diagnostic (see section 2.3 for details) in TCV is about $1.5 \mathrm{~m}$, which is comparable to the path length of the edge-to-resonance path in ITER. The versatility of TCV enabled the development of an SMT plasma scenario [8].

\subsection{Simple magnetized toroidal plasmas in TCV}

In the SMT configuration [2], a vertical magnetic field $B_{v}$ is imposed on a toroidal magnetic field field $B_{T}$ resulting in helical open field lines with $\nabla B$ and curvature, terminating on the upper and lower wall of the TCV vessel. The plasma is generated and sustained by the injection of mmpower at the second harmonic of the electron cyclotron resonance frequency (X2). In this configuration, plasma turbulence is dominated by interchange instabilities, characterized by field-aligned elongated structures - referred to as blobs - with $k_{\|}<<k_{\perp}\left(k_{\|}\right.$and $k_{\perp}$ being the parallel and the perpendicular wave number respectively) [2]. Blobs are formed in the crest of an interchange wave, and their internal electric field gives rise to an $\mathbf{E} \times \mathbf{B}$ radial motion. Fluctuations of electron density $n_{e}$ can reach up to $100 \%$ of the time-averaged density.

Line-integrated density is measured inside the vessel using the Far Infrared Interferometer [9]. Biased ( $-100 \mathrm{~V}$ bias) wall-mounted Langmuir Probes (LP) measure the ion saturation current, which is proportional to the electron den-

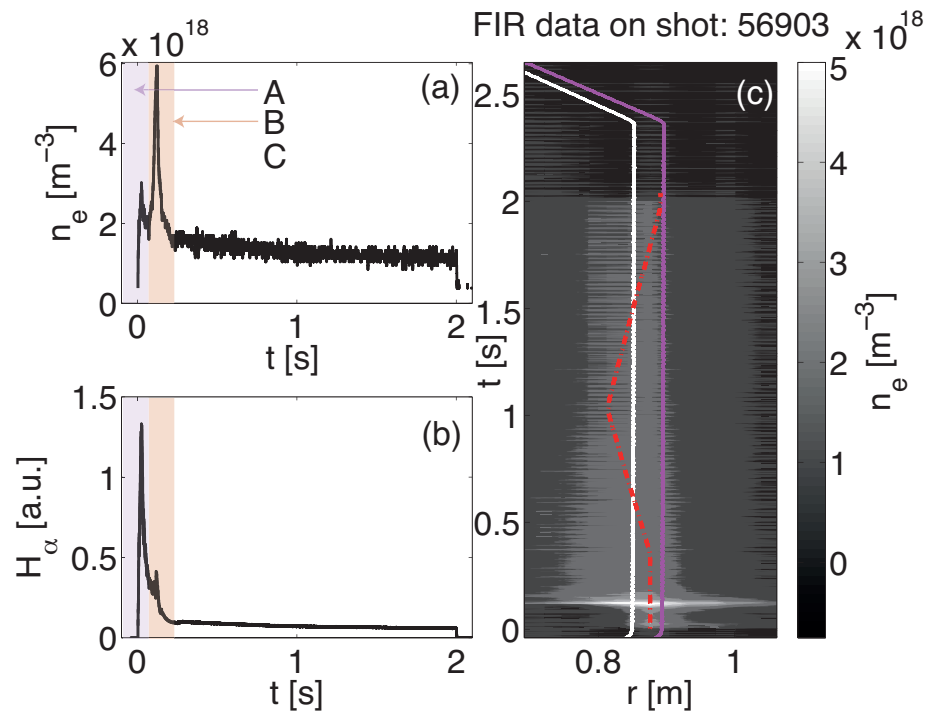

Figure 2. A typical SMT discharge in TCV: $D_{2}$ gas is ionized by the injection of mm-power at the second electron cyclotron harmonic in X-mode (phase A, (a) and (b)). During this phase, the plasma is localized around the beam. The second phase (B) is a transition phase towards a steady-state, EC-sustained, SMT (phase C). (c) : Time evolution of the line integrated electron density measured by the FIR. The X2 cold resonance is represented in white and the $\mathrm{X} 3$ cold resonance in magenta. The red dotted line is a visual aid : it represents the radial position of the $\mathrm{X} 3$ beam at the floor of the machine, with no refraction in the case of a double sweep of the launching mirror poloidal angle $\theta_{m}$

sity, at the edge of the vessel [10].

Breakdown in SMT configuration is achieved in TCV by the injection of $450 \mathrm{~kW} \mathrm{X2} \mathrm{mm-power} \mathrm{in} \mathrm{the} \mathrm{vessel} \mathrm{filled}$ with $D_{2}$ gas, Fig. 1. The first breakdown phase (phase A) lasts about $0.1 \mathrm{~s}$, see Fig. 2. This phase corresponds to the ionisation of the plasma. Images from a fast framing camera shows that during this phase, the plasma is locally formed around the X2 beam. In a second phase (phase B) (typically from 0.1 to $0.2 \mathrm{~s}$ ), the plasma is spread throughout the vessel and reaches a stationary phase (phase C). During this phase, electron densities are typically between 0.7 and $4 \times 10^{18} \mathrm{~m}^{-3}$ and electron temperatures between 30 and $50 \mathrm{eV}$. The duration of the plasma was only limited by the gyrotron pulse length, and could in principle be extended to $4 \mathrm{~s}$ using back-to-back pulses, as previously done for fully Electron Cyclotron Current Driven (ECCD) plasmas [7].

\subsection{X3 Transmission Diagnostic (X3TD)}

An X3 beam is launched from the top of the vessel using a steerable mirror. Sweeping the mirror poloidal angle $\theta_{m}$ in vacuum enables the reconstruction of the beam poloidal profile [11]. The power reaching the floor of the vessel is coupled to a corrugated $H E_{11}$ graphite waveguide after $-55 \mathrm{~dB}$ attenuation. The attenuation is achieved by an hexagonal array of 241 circular holes with diameter $d=1.2 \mathrm{~mm}$ such that the cut-off frequency is $f_{c}=146.4 \mathrm{GHz}$. The attenuation waveguide is placed 
inside the vacuum vessel to protect the sapphire window that provides the vacuum feedthrough. The sapphire window is matched for a minimum of reflection at $118 \mathrm{GHz}$. The transmission of the attenuation cap is independent of its orientation around the waveguide, therefore no precaution is needed during the installation. An additional $20 \mathrm{~dB}$ attenuation is introduced by an absorbing foam placed within the $H E_{11}$ waveguide that is attached to the sapphire window outside of the vacuum vessel. The $H E_{11}$ mode is then coupled to a $T E_{10}$ mode in a WR6.5 rectangular waveguide through a special Gaussian Optical Lens-like Antenna (GOLA). The mm-power is measured by a Schottky diode and digitally acquired at $250 \mathrm{kHz}$ acquisition frequency.

\subsection{Experimental challenges}

The SMT experimental campain on TCV was experimentally challenging. We found that the plasma parameters are sensitive to wall conditions. Therefore operation conditions are to be predetermined for each experimental session.

Another challenge is due to the nature of the experiment itself. High power mm-power is launched in the vacuum vessel with no first-pass absorption from the plasma. Direct reflections at the floor of the vessel must be avoided. They can destabilize the operation of the gyrotron by causing arcs or mode losses. TCV is also equipped with an extensive stray radiation protection system. The signals of the stray detectors are combined with a comparison between the expected and actual electron density to determine if stray radiations are potentially hazardous to other components and if so stop the discharge. Good knowledge of beam propagation inside the vessel is therefore required for successful transmission.

\section{Results}

In this section, we present the first measurements of the beam profile through plasma density fluctuations. Previous investigations of $\mathrm{X} 3$ beam profiles and absorption in TCV in vacuum are presented in [11] and show that the beam profile is Gaussian as expected in the design.

\subsection{First measurements of beam profiles through plasma}

X3 power $P_{X 3}$ is injected (Fig. 3) after the plasma breakdown (phase A and B, Fig. 2) through the SMT plasma with no sign of absorption. A gyrotron delivers constant power for $1.7 \mathrm{~s}$ Fig. 3. It takes about $0.5 \mathrm{~s}$ to $0.6 \mathrm{~s}$ for the gyrotron frequency to stabilize Fig. 3 a). as indicated by significant fluctuations in the forward power measurement in the transmission line (due to mode beating). The nature of the absorption cap of the X3TD makes it frequency sensitive. Analysis of the mm-transmitted power can thus not be performed on the first part of the discharge in Fig. 3. Unless very short pulses are used, this imposes a minimum
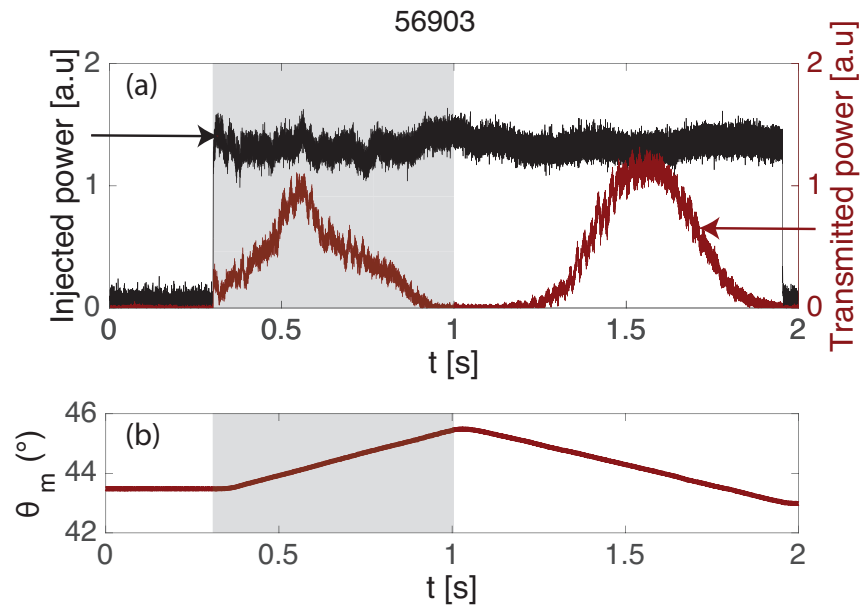

Figure 3. A double sweep of the X3 beam across the transmission diagnostic (a) is performed by sweeping the poloidal angle $\theta_{m}$ of the launching mirror (b). (a) shows an asymetry between the received powers. The stabilization time of the gyrotron frequency is identified in gray.

duration for plasma discharges.

Fig. 4 shows the beam profile for two different plasma electron densities $n_{e}=2.3 \times 10^{18} \mathrm{~m}^{-3}$ and $n_{e}=$ $3.4 \times 10^{18} \mathrm{~m}^{-3}$. Strong fluctuations of the transmitted power (up to $70 \%$ of the peak power) are caused by the fluctuations $\delta n_{e}(\mathbf{x}, t)$ of the electron density. The correlation between $\delta n_{e}(\mathbf{x}, t)$ and fluctuations of the transmitted power $\delta P_{T, X 3}(\mathbf{x}, t)$ are investigated in the next section. Influence of the electron density on the beam propagation is under investigation. Refraction effects tied to the electron density are visible in Fig. $4:$ as the launcher and receiver location are fixed in space a higher electron density requires a larger $\theta_{m}$ to compensate for refraction. Work is under way to estimate the contribution of the refractive effect to the measured beam profile, as well.

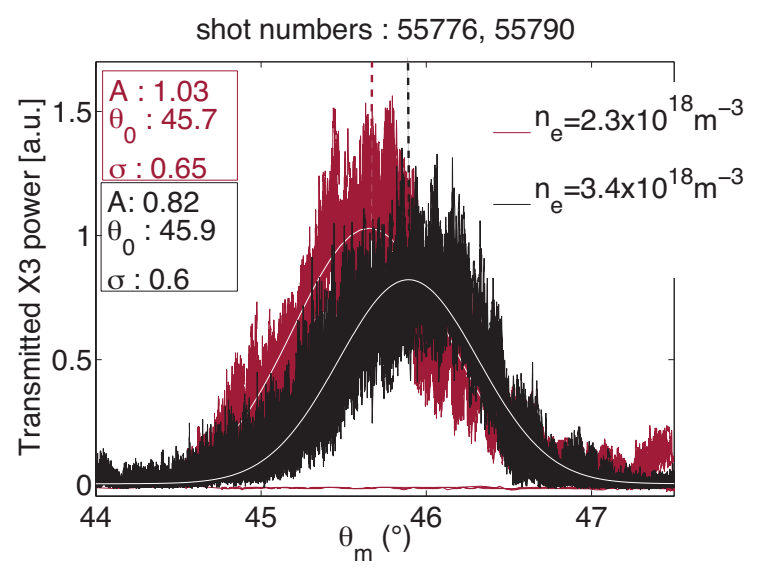

Figure 4. Transmitted mm-power for two different plasma electron densities. The large fluctuations of the signal are dominated by electron density fluctuations. The peak is displaced by refraction. Top-left parameters are the fitting parameters of the mm-signal to a Gaussian of the form: $A_{0} e^{-\left(\frac{\theta_{m}-\theta_{0}}{\sigma}\right)^{2}}$ 


\subsection{Statistical analysis of transmitted power and electron density fluctuations}

Analysis of the transmitter mm-power signal has shown a $10 \%$ correlation with the electron density fluctuations measured by Langmuir probes located at the TCV floor. Results are shown in Fig. 5.
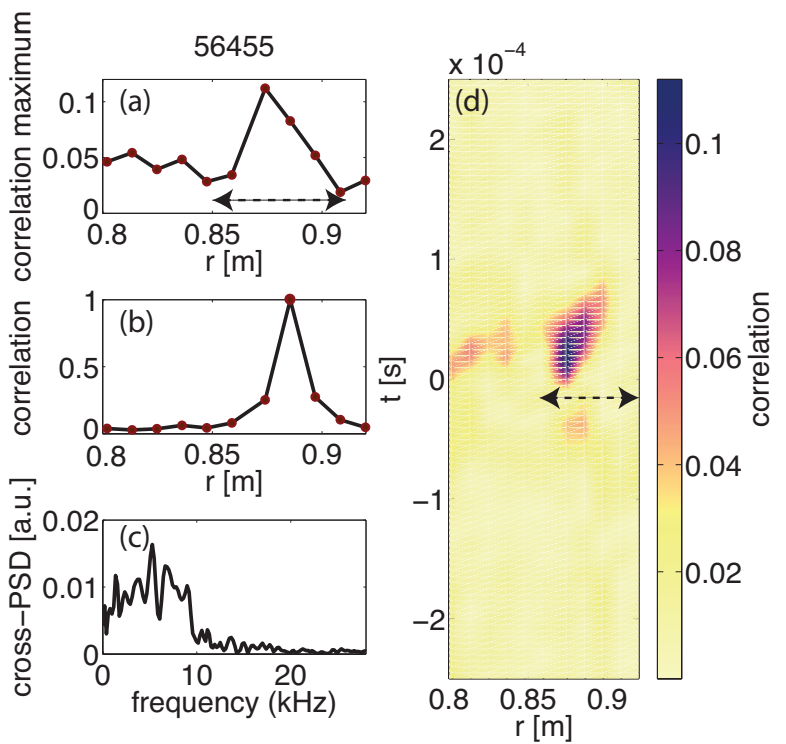

Figure 5. (a) and (d): correlation between the mm-power transmitted signal and the floor Langmuir probes. The black-dotted arrow shows the extent of the $H E_{11}$ waveguide of the X3TD. (b): Correlation between a LP facing the $H E_{11}$ waveguide and the neigbouring probes. (c) Cross power spectral density between a LP facing the $H E_{11}$ waveguide and the transmitted mm-power, most of the information between the two signals is exchanged between 0 and $10 \mathrm{kHz}$. (d) The time scale is derived from $\mathrm{m}$ in equation (1).

To avoid confusion between several definitions of the correlation function found in literature, we define the one used in this paper. The computation of the crosscorrelation is the following :

Let $x$ and $y$ be two discrete signals of length $N, f$ and $g$ are the corresponding standardized functions $\left(f_{i}=\frac{x_{i}-<x>}{\sigma_{x}}\right.$ and $\left.g_{i}=\frac{y_{i}-<y>}{\sigma_{y}}\right)$. The cross-correlation function $\hat{R}_{x y}$ is given by:

$$
\hat{R}_{x y}(m)=\left\{\begin{array}{r}
\frac{1}{N} \sum_{n=0}^{N-m-1} f_{n+m} g_{n}, m \geq 0 \\
\hat{R}_{x y}(-m), m<0
\end{array}\right.
$$

where $m=0,1, \ldots, 2 N-1$. The correlation function has a maximum value if 1 if $x=y$ and is zero if $x$ and $y$ are uncorrelated. For a signal sampled at the frequency $f_{s}$, we can convert $m$ in time units as follow: $t(m)=\frac{1}{f_{s}}(N-m)$ Fig. 5 shows that the LP signals exhibiting the best correlation with the transmitted mm-power signal are the LP probes which are located at the same radial position as the X3TD $H E_{11}$ waveguide. The information between them is mostly shared at frequencies ranging between 0 and $10 \mathrm{KHz}$ (Fig. 5 (c)). In addition, the spatial scale of the turbulent edge structures is obtained by computing the correlation between one LP located at the floor of the vessel and the neighboring probes. The correlation indicates the presence of turbulent structures with a spatial scale length typically in the order of $2 \mathrm{~cm}$ as shown in Fig. $5 \mathrm{~b}$ ).

\section{Summary and outlook}

The effect of plasma edge turbulence on the propagation of a high-power mm-beam is investigated in TCV. A plasma scenario in the simple magnetized toroidal configuration is developed in TCV. Steady-state plasmas are obtained and sustained by the injection of mm-power at the second electron cyclotron frequency. Several electron densities are achieved. A high-power diagnostic is installed in TCV to measure the $\mathrm{X} 3$ beam profile after passing through the plasma turbulence. The mm-power fluctuations are shown to be correlated with edge measured electron density fluctuations. Methods to vary the fluctuation level along the beam trajectory are presently investigated; e.g. by moving the source region relative to the X3TD by changing the magnetic field. A numerical effort is also under way to compare experimental measurements with WKBeam [3], GRAY [12] and IPF-FDMC [6].

\section{Acknowledgements}

One of the authors, O. Chellaï, would like to gratefully thank Basil P. Duval, Antoine Merle and Miguel Silva for the interesting discussions and advices during the experimental sessions. This work has been carried out within the framework of the EUROfusion Consortium and has received funding from the Euratom research and training programme 2014-2018 under grant agreement No 633053. The views and opinions expressed herein do not necessarily reflect those of the European Commission.

\section{References}

[1] O. Sauter, et al., Plasma Physics and Controlled Fusion 52, 025002 (2010)

[2] I. Furno, et al., Physical Review letters 100, 055004 (2008)

[3] H. Weber, et al., in EPJ Web of Conferences (EDP Sciences, 2015), Vol. 87, p. 01002

[4] C. Tsironis, et al., Physics of Plasmas 16, 112510 (2009)

[5] A. Rham, et al., Physics of Plasmas 20, 056110 (2013)

[6] A. Köhn, et al., Plasma Physics and Controlled Fusion 58, 105008 (2016)

[7] S. Coda, et al., Nuclear Fusion 51, 094017 (2011)

[8] A. Fasoli, et al., Physics of Plasmas 13, 055902 (2006)

[9] S. Barry, et al., in Proceedings of the 8th international symposium on Laser-aided Plasma Diagnostics (LAPD-8) (1997) 
[10] L. Tonks, et al., Physical Review 34, 876 (1929)

[11] T. Goodman, et al., in EPJ Web of Conferences (FEC, IAEA, 2016)
[12] D. Farina, et al., Fusion Science and Technology 52, 154 (2007) 